\title{
ELASTIC VIBRATIONS OF PLANAR AND DETERMINISTIC ROUGH SURFACES
}

\author{
B. Djafari Rouhani, A. Khelif, E.H. El Boudouti*, A. Akjouj \\ AND L. DOBRZYNSKI \\ Laboratoire de Dynamique et Structure des Matériaux Moléculaires, associé au \\ CNRS, UFR de Physique, Université des Sciences et Technologies de Lille \\ 59655 Villeneuve d'Ascq, France
}

We present a few recent or new theoretical results about surface acoustic localized and resonant modes associated with planar or deterministic rough surfaces. The following composite systems are considered: one or two adlayers deposited on a semi-infinite substrate and wires or grooves integrated near a planar surface. The surface modes can be obtained as well-defined features of the density of states resulting from a calculation of the Green functions in these heterostructures. In this work, the materials are described in the framework of the elasticity theory.

PACS numbers: $68.35 . \mathrm{Ja}, 68.45 . \mathrm{Kg}$

\section{Introduction}

The acoustic vibrations of a homogeneous planar surface are modified in presence of surface inhomogeneities such as adlayers or microstructures integrated onto the solid surface. The object of this paper is to discuss these effects in a few heterostructures where the symmetry of translation parallel to the surface is either conserved (like for an adlayer or a multilayer grown on a substrate) or broken (as in the case of a rough surface containing a ridge or a groove). Throughout this paper, the constituent materials in each heterostructure will be described in the framework of elasticity theory and assumed, for the sake of simplicity, to be isotropic. Among different mathematical approaches, the Green function method is quite suitable for studying the spectral and scattering properties of these composite materials; in particular, it enables us to calculate the total or local densities of states (DOS) in which the localized or resonant surface modes appear as well-defined peaks. The next two sections will respectively be devoted to the presentation of the vibrational properties in the following geometries: (i) a layer or a bilayer grown on a substrate, and (ii) a wire deposited on a planar surface.

\footnotetext{
*Also at: Département de Physique, Faculté des Scciences, Oujda, Marocco.
} 


\section{Adlayer, bilayer and multilayer on a substrate}

A semi-infinite isotropic elastic medium bounded by a stress-free planar surface can support localized surface vibration modes, the well-known Rayleigh waves [1]. These modes are of sagittal polarization which means that the displacement field lies in the sagittal plane defined by the wave vector $k^{\prime \prime}$ (or the propagation direction) parallel to the surface and the normal to the surface.

In presence of an isotropic adlayer, the surface vibrational modes also include Love and Sezawa waves, respectively of shear horizontal and sagittal polarizations, which have the character of standing waves in the slab with their amplitude decaying exponentially with increasing distance into the substrate from the interface [1]. These waves are defined in the case the transverse velocity of sound in the adsorbate $C_{t 1}$ is lower than that in the substrate $C_{t s}$ (see Figs. 1 and 2): with increasing $k^{\prime \prime}$, they emerge from the bottom of the substrate bulk bands (defined
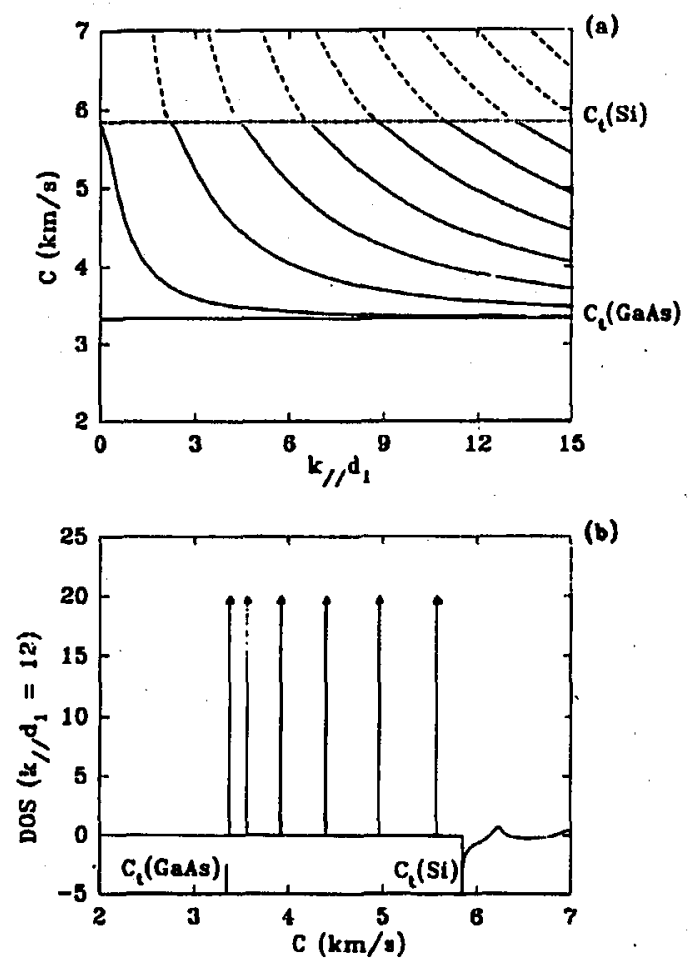

Fig. 1. (a) Dispersion curves of localized (full curves) and resonant (dashed curves) transverse modes for a GaAs slab of thickness $d_{1}$ on a Si substrate. $C$ is the velocity. (b) Variation of the density of states (in units of $d_{1} / C_{t}(\mathrm{GaAs})$ ) due to the adsorption of the GaAs slab, at $k^{\prime \prime} d_{1}=12$. The arrows indicate the delta peaks associated with the localized (Love) modes. 

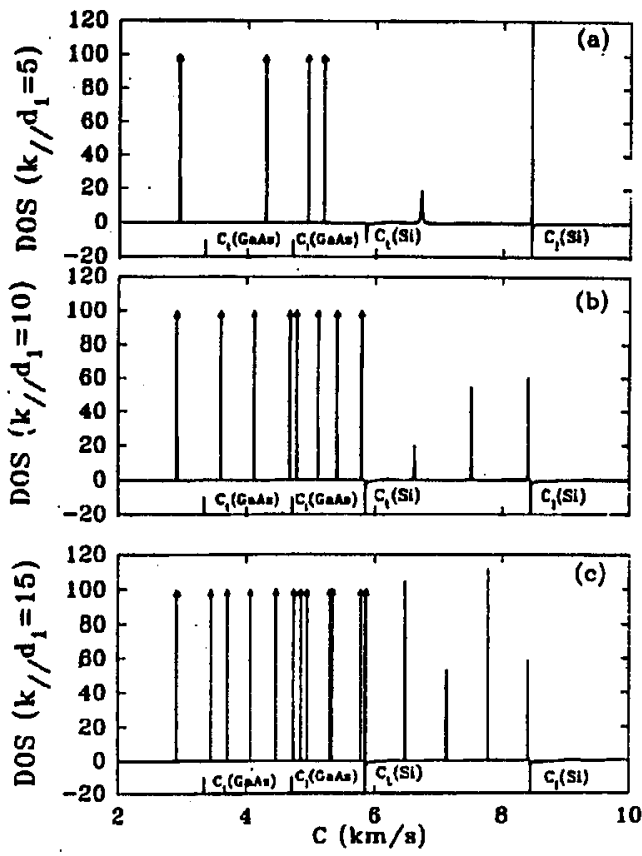

Fig. 2. Variation of the density of states of the sagittal modes due to the adsorption of a GaAs slab on a Si substrate, at $k^{\prime \prime} d_{1}=5(\mathrm{a}) ; 10$ (b); 15 (c). The arrows indicate the delta peaks associated with the localized (Sezawa) modes.

by $\omega / k^{\prime \prime}=C_{t s}$, where $\omega$ is the frequency), their dispersion being a function of the quantity $k^{\prime \prime} d_{1}$, where $d_{1}$ is the thickness of the adlayer. Their extensions into the substrate bulk bands (i.e. $C=\omega / k^{\prime \prime}>C_{t s}$ ) correspond to resonant modes, also called leaky or pseudo-waves, radiating their energy into the bulk modes and therefore having finite lifetimes. However, it has been evidenced both theoretically and experimentally $[2,3]$ that some of these waves can take the character of longitudinal guided waves in the slab when their velocities lie below the longitudinal velocity of sound in the substrate $C_{l s}$ : despite a coupling to propagating transverse modes, their lifetimes may become comparable to that of Rayleigh or Sezawa modes. Brillouin scattering of these modes has been reported in $\mathrm{ZnSe}$ films on GaAs or in a polymeric Langmuir-Blodgett film on molydenum, together with a theoretical analysis of light scattering cross-section $[2,3]$.

In the opposite case of $C_{t 1}>C_{t s}$, the modifications induced by the adlayer in the vibrational spectra can only appear as resonant or pseudo-modes falling inside the substrate bulk bands. In any case, the dispersion and lifetime of these waves are dependent upon the parameter $k^{\prime \prime} d_{1}$, in addition to the material parameters.

We have developed [4] an analysis of the above localized and resonant surface modes within a Green function formalism which enables us to calculate both local and total DOS: localized modes appear as delta peaks outside the substrate bulk bands, whereas the positions and widths of the peaks inside these bands give 
the frequencies and lifetimes of the resonant modes which may be more or less pronounced features depending on material parameters and on $k^{\prime \prime} d_{1}$. An example of this calculation is presented in Figs. 1 and 2 for a GaAs layer on a Si substrate, respectively for shear horizontal and sagittal polarizations. It is worth pointing out that the intensities of the resonant peaks are very dependent upon the elastic parameters of the substrate and adsorbate materials (see Ref. [4]); for example, the transverse resonant modes of Fig. 1 are actually very weak.

Our analysis can also be applied to the case of a bilayer or a multilayer grown on a substrate. Here, we consider the problem of a bilayer, as in recent works $[5,6]$ where the surface modes and pseudo-modes of a $\mathrm{Si}_{-}-\mathrm{SiO}_{2}$ bilayer on a Si substrate were investigated both theoretically and by Brillouin experiments. Discussing all possible combinations of material parameters or velocities is outside the scope of this paper; instead, we would emphasize one particular result, not reported before to our knowledge, and illustrated here in Figs. 3 and 4 for a GaAs-Si bilayer on a GaAs substrate: this is the possibility of finding well-defined guided pseudo-modes in the top layer, as a consequence of its separation from the substrate by the buffer layer, when the velocities of sound in the buffer are higher
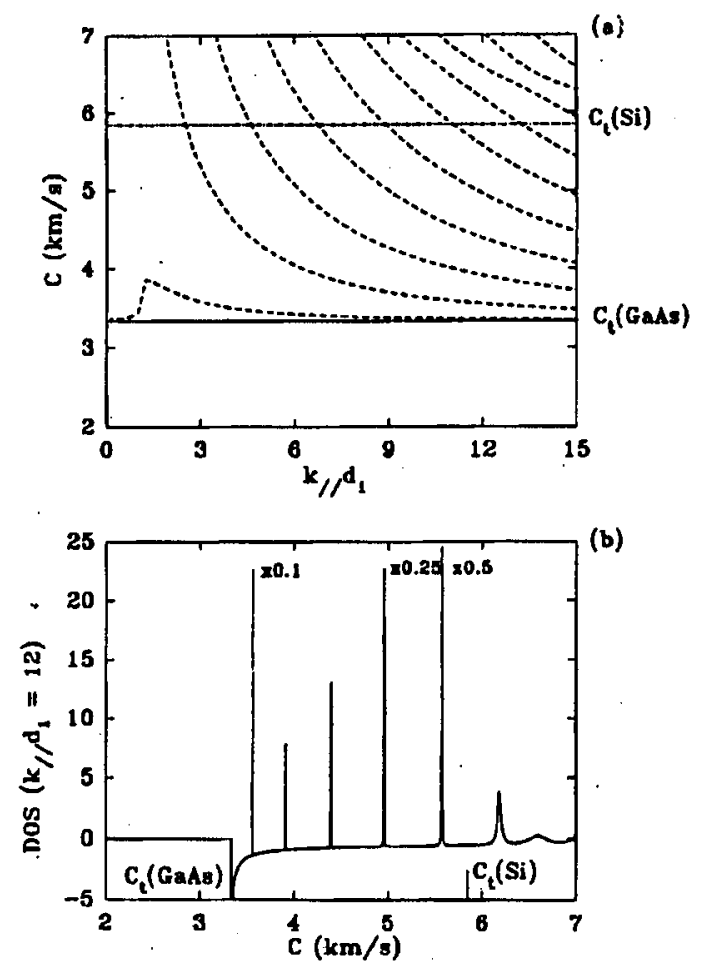

Fig. 3. Same as in Fig. 1 for a GaAs-Si bilayer on a GaAs substrate. The figure is sketched for $d_{2}=d_{1} / 2$, where $d_{1}$ and $d_{2}$ are respectively the thicknesses of the GaAs and Si slabs. 


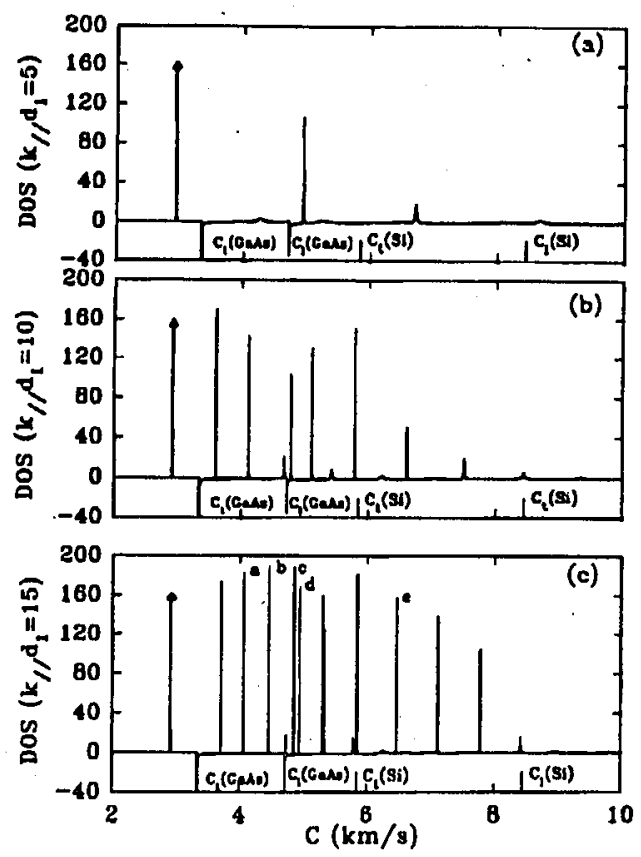

Fig. 4. Same as in Fig. 2 for a GaAs-Si bilayer on a GaAs substrate. In part (c), the height of the peaks labelled $a, b, c, d, e$ are respectively multiplicd by $0.07,0.3,0.8,0.15$ and 0.5 .

than those in the topmost layer. This phenomenon can be observed for both shear horizontal (Fig. 3) and sagittal (Fig. 4) polarizations; it results from the fact that some of the slab modes of the top layer, even if they are in resonance with the substrate bulk modes, cannot propagate in the intermediate layer and therefore remain well-defined guided waves of the higher slab. Indeed, in the velocity range below $C_{t}(\mathrm{Si})$, one can observe both in Figs. 3 and 4 very narrow resonant modes corresponding to guided waves of the topmost GaAs slab even though they are in resonance with the bulk modes of the GaAs substrate. These peaks in the DOS broaden, and therefore the localization of the corresponding modes decreases, when either the thickness of the buffer layer $d(\mathrm{Si})$ or the quantity $k^{\prime \prime} d(\mathrm{GaAs})$ decrease. In Fig. 4 there are also well-defined resonances with velocities falling between $C_{t}(\mathrm{Si})$ and $C_{l}(\mathrm{Si})$ which are guided modes of the whole GaAs-Si bilayer.

Finally, to end this section, we would mention our study of localized and resonant elastic waves associated with the surface of a superlattice (composed of a periodic repetition of two different materials) or with a superlattice-substrate interface [7].

\section{Adsorbed wire on a planar surface}

The integration of well-defined deterministic microstructures onto a solid surface has become possible due to the progress in advanced fabrication techniques such as microlithography or molecular beam epitaxy. The spectral and response 
properties of these structures as well as their imaging are of current interest. One can mention the investigation of surface acoustic shape resonances [8-10] or the scattering of acoustic waves by single resonating elements [11-14] and by periodic or quasi-periodic [15] solid surfaces. In Ref. [10] we have developed an exact numerical method to calculate the Green function and the dynamical properties of a wire (or a groove) of arbitrary shape near a planar surface. In particular, we presented general formula to obtain the variation in the total DOS due to the presence of the wire and the scattering of an incoming wave by this surface defect; these expressions only involve the matrix elements of the Green function in the reduced space of the wire-substrate interface. Although the general theory can be applied to any type of excitations or any polarization of the vibration modes, we limited ourselves to the simplest case of shear horizontal vibrations for which the displacement field has a single component parallel to the direction of the wire. This is because the Green function of the perfect bulk or semi-infinite solid are analytically known in this case. In Ref. [10] we were interested in acoustic shape resonances of an adsorbed wire; here, we also present an application of our formalism to the scattering of an acoustic plane wave by such a surface defect.

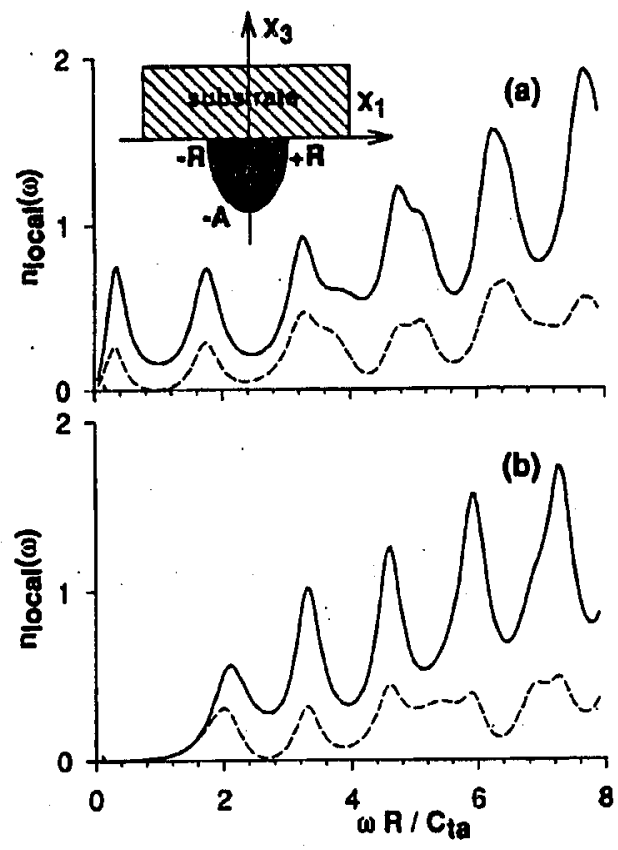

Fig. 5. Symmetric (a) and antisymmetric (b) modes associated with an adsorbed wire over a substrate. Full lines: average local density of states along the free surface of the wire (in units of $R / C_{t a}$ ) as a function of $\omega R / C_{t a}$; dashed lines: average local density of states along the planar interface between the wire and the substrate. The inset shows the geometry of the problem. 
In the following illustrations we assume that the wire is of parabolic shape and defined by the equation (see the inset of Fig. 5): $\zeta\left(x_{1}\right)=-A\left[1-\left(x_{1} / R\right)^{2}\right]$ for $x_{1} \in[-R, R]$; the height $A$ of the parabola is taken to be $A=2 R$. We also use the following set of parameters for the wire and substrate materials, labelled respectively as $a$ and $b: C_{t a}=2 C_{t b}$ and $\rho_{a}=\rho_{b}$ (where $\rho$ is the mass density).

Owing to the symmetry of the wire, the acoustic resonances may be distinguished between symmetric (S) and antisymmetric (AS) modes. Figure 5 shows, for both symmetries, the average local DOS at the free surface of the wire and at the wire-substrate interface. One can first notice the existence of well-defined resonances in these local DOS which actually happen at the same frequencies as the peaks in the total DOS and also show a good correspondence with the eigenmodes of an isolated wire having its boundary free of stress [10]. However, it should be pointed out that for other material parameters [10] the peak positions in the total DOS and in the local DOS at the wire-substrate interface may be quite different and these peaks may also be significantly shifted with respect to the eigenfrequencies of the isolated wire.

Let us now consider a plane wave with frequency $\omega$ travelling towards the surface with an incident angle $\theta_{i}$. The total reflected field can be decomposed into a first component representing the reflection from a perfect planar surface, and

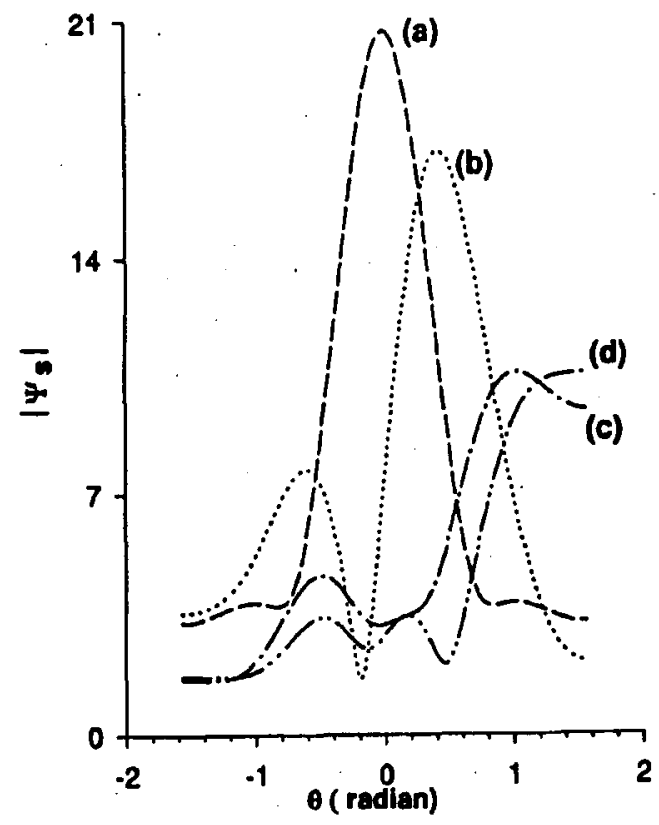

Fig. 6. Amplitude of the scattered field $\left|\Psi_{s}\right|$ as a function of the polar angle $\theta$, at a distance $r=4 R$ of the origin, for different incident angles $\theta_{\mathrm{i}}=0(\mathrm{a}) ; \pi / 6(\mathrm{~b}) ; \pi / 3$ (c) and $\pi / 2(\mathrm{~d})$. The frequency is taken to be $\omega=2.5 C_{t a} / R$. 
a second component $\Psi_{s}$ describing the diffuse (or scattered) part of the wave we shall discuss in the following.

The amplitude $\left|\Psi_{\mathrm{s}}\right|$ of the scattered field presents important variations with the frequency of the incident wave, with a succession of enhancement and decrease; at normal incidence where the symmetric modes only participate to the scattering, $\left|\Psi_{s}\right|$ almost vanishes at some frequencies corresponding to symmetric eigenmodes of an isolated wire with free stress boundary conditions, i.e. at those frequencies where the presence of the wire does not perturb the boundary condition at the planar surface of the substrate. The angular distribution of $\left|\Psi_{s}\right|$ is also dependent upon the frequency and upon the incident angle. As an example, we have presented in Fig. 6, for different incident angles $\theta_{\mathrm{i}}$, the amplitude of the scattered field as a function of the polar angle $\theta$, at a distance $r=4 R$ of the origin and for a frequency $\omega=2.5 C_{t a} / R$ : at normal incidence, $\left|\Psi_{\mathrm{s}}\right|$ is exactly symmetrical with respect to $\theta$; for an oblique incidence, it may present a major wing in the forward direction and a minor wing in the backward direction or even several weak oscillations.

More detailed results about the behaviour of the far field and near field scattered wave will be presented in a forthcoming paper. As a general remark, one can point out the interest of the Green function approach in calculating both the spectral and scattering properties of surface defects. The method used here can also be applied to the study of other types of excitations.

\section{References}

[1] For a general review of surface acoustic waves, see A.A. Maradudin, in: Nonequilibrium Phonon Dynamics, Ed. W. Bron, Plenum, New York 1985, p. 395; G.W. Farnell, E.L. Adler, in: Physical Acoustics Principles and Methods, Vol. 9, Eds. W.P. Mason, R.N. Thurston, Academic, New York 1972, p. 35.

[2] B. Hillebrands, S. Lee, G.I. Stegman, H. Cheng, J.E. Potts, F. Nizzoli, Phys. Rev. Lett. 60, 832 (1989); Surf. Sci. 211/212, 387 (1989).

[3] F. Nizzoli, B. Hillebrands, S. Lee, G.I. Stegman, G. Duda, G. Wegner, W. Knoll, Phys. Rev. B 40, 3323 (1989).

[4] A. Akjouj, E.H. El Boudouti, B. Djafari Rouhani, L. Dobrzynski, J. Phys., Condens. Matter 6, 1089 (1994).

[5] F. Nizzoli, C. Byloos, L. Giovannini, C.E. Bottani, G. Ghislotti, P. Mutti, Phys. Rev. B 50, 2027 (1994); G. Ghislotti, C.E. Bottani, Phys. Rev. B 50, 12131 (1994).

[6] C. Byloos, L. Giovannini, F. Nizzoli, Phys. Rev. B 51, 9867 (1995); G. Ghislotti, C.E. Bottani, P. Mutti, C. Byloos, L. Giovannini, F. Nizzoli, Phys. Rev. B 51, 9875 (1995).

[7] For a review of vibrations in superlattices, see J. Sapriel, B. Djafari Rouhani, Surf. Sci. Rep. 10, 189 (1989). More recently, we have studied the density of states of acoustic waves in semi-infinite superlattices and given general rules about the existence of surface states in: B. Djafari Rouhani, E.H. El Boudouti, E.M. Khourdif, Vacuum 45, 341 (1994); E.H. El Boudouti, B. Djafari Rouhani, E.M. Khourdifi, L. Dobrzynski, Phys. Rev. B 48, 10987 (1993); E.H. El Boudouti, B. Djafari Rouhani, A. Nougaoui, Phys. Rev. $B 51,13801$ (1995). The case of a finite superlattice deposited on a substrate has been investigated in E.H. El Boudouti, B. Djafari Rouhani, Phys. Rev. B 49, 4586 (1994).

[8] A.A. Maradudin, P. Ryan, A.R. Mc Gurn, Phys. Rev. B 38, 3068 (1988). 
[9] B. Djafari Rouhani, A.A. Maradudin, Solid State Commun. 73, 173 (1990).

[10] B. Djafari Rouhani, L. Dobrzynski, J. Phys., Condens. Matter 5, 8177 (1993);

B. Djafari Rouhani, L. Dobrzynski, A. Khelif, Prog. Surf. Sci. 48, 301 (1995).

[11] E. Danicki, Wave Motion 9, 445 (1987).

[12] N.S. Clarke, C. Davies, J.S. Burdess, Wave Motion 11, 371 (1989).

[13] A.A. Maradudin, T. Michel, B. Djafari Rouhani, A.R. Mc Gurn, A.R. Baghai-Wadji, in: Continuum Models and Discrete Systems, Vol. I, Ed. G.A. Maugin, Longman, Essex 1990, p. 227.

[14] V.P. Plessky, N.N. Shibanova, in: Proc. Ultrasonics Int., 1991, p. 115.

[15] L. Macon, J.P. Desideri, D. Sornette, Phys. Rev. B 44, 6751 (1991). 